\title{
Aspectos clínicos, laboratoriais e cirúrgicos de 15 casos de piometra em cadelas
}

\author{
Clinical, laboratorial and surgical aspects of 15 cases of pyometra in bitches \\ Aspectos clínicos, de laboratorio y quirúrgicos de 15 casos de piometra em perras
}

Recebido: 07/07/2021 | Revisado: 13/07/2021 | Aceito: 20/07/2021 | Publicado: 28/07/2021

\author{
Lucas Ariel Rossi \\ ORCID: https://orcid.org/0000-0002-3283-1605 \\ Centro Universitário da Serra Gaúcha, Brasil \\ E-mail: lucasarielrossi@ hotmail.com \\ Manoela Maria Bianchi \\ ORCID: https://orcid.org/0000-0003-3624-6486 \\ Centro Universitário da Serra Gaúcha, Brasil \\ E-mail: vet.manoela@gmail.com \\ Letícia da Silva \\ ORCID: https://orcid.org/0000-0002-6146-4912 \\ Centro Universitário da Serra Gaúcha, Brasil \\ E-mail: letisilva@hotmail.com \\ Carolina da Fonseca Sapin \\ ORCID: https://orcid.org/0000-0002-2757-6355 \\ Centro Universitário da Serra Gaúcha, Brasil \\ E-mail: carolina.sapin@fsg.edu.br
}

\begin{abstract}
Resumo
A piometra é uma doença caracterizada pela infecção supurativa do útero, é uma doença mediada por hormônios e pode ocorrer em qualquer fase do ciclo estral, porém tem maior predisposição na fase do diestro. O objetivo deste trabalho foi realizar um estudo comparativo entre quinze casos de piometra em cadelas atendidas em uma clínica veterinária de Caxias do Sul. Foi feita uma análise de quinze prontuários de cadelas diagnosticadas com piometra, onde foram coletados e avaliados dados clinico-epidemiológicos como raça, porte, idade, histórico clínico, sinais clínicos, exames complementares e classificação da piometra, assim como o tratamento de escolha. Neste estudo houve uma predominância de piometra fechada em relação à piometra aberta. Os sinais clínicos mais encontrados foram anorexia, prostração e secreção vulvar. É importante ressaltar que exames complementares de sangue e imagem devem ser realizados para a elucidação de cada caso, tendo a ovariohisterectomia como método definitivo de tratamento.
\end{abstract}

Palavras-chave: Hiperplasia endometrial cística; Trato reprodutivo; Progesterona.

\begin{abstract}
Pyometra is a disease characterized by an supurative infection in the uterus, it is a disease that has hormones influence and can occur at any phase of the estrous cycle, but has a bigger predisposition in the diestrous. The objective of this paper was na comparative study between fifteen medical records of bitches diagnosed with pyometra. The epidemiologic datas such as breed, size, age, medical history, clinical sings, complementary exams, pyometra type and treatment of choice were colected and rated. In this study closed pyometra was the predominant type. The most prevalente clinical signs were anorexia, prostration and vulvar secretion. It is important to highligt that the blood and images exams were done to clarify each case and the ovariohisterectomy was the treatment of choice.
\end{abstract}

Keywords: Cystic endometrial hyperplasia; Reproductive tract; Progesterone.

\section{Resumen}

La piometra es una enfermedad caracterizada por una infección supurativa del útero, es una enfermedad mediada por hormonas y puede ocurrir en cualquier etapa del ciclo estral, pero es más propensa a la fase del diestro. El objetivo de este trabajo fue realizar um estúdio comparativo entre quince casos de piometra en perras atendidas en una clinica veterinaria en Caxias do Sul. Se realizó un análisis de quince historias clinicas de perras diagnosticadas con piometra donde se encontraron datos clinico-epidemiologicos como la raza. Se recogieron y evaluaron tamaño, edad, historia clinica, signos clínicos, exámenes complementários y clasificación de la piometra, así como el tratamento de elección. En este estudio, hubo un predominio de piometra cerrada sobre piometra abierta. Los signos clínicos más frecuentes fueron anorexia, prostración y secreción vulvar. Es importante destacar que se realizan pruebas complementarias de sangue y de imagen para el esclarecimiento de cada caso, siendo la ovariohisterectomia el método definitivo.

Palabras clave: Hiperplasia endometrial quistica; Tracto reproductivo; Progesterona. 


\section{Introdução}

O complexo hiperplasia endometrial cística - piometra caracteriza-se por ser um distúrbio uterino frequente em cadelas e potencialmente fatal (Nelson; Couto, 2015). A progesterona estimula o crescimento e a atividade das glândulas endometriais, o que pode resultar em um acúmulo de líquidos na luz uterina. Devido à redução da contratilidade miometrial, ocorre um favorecimento a infecções bacterianas secundárias no útero (Ettinger; Feldman, 2004). As bactérias que invadem o útero são oriundas da vulva/vagina (Nelson; Couto, 2015). A mais comumente encontrada em casos de piometra é a Escherichia coli (Fossum et al., 2019).

A piometra pode ser classificada como de cérvix aberta ou fechada. Classifica-se como aberta quando há presença de corrimento vulvar, e como fechada quando o útero se encontra palpável, aumentado de tamanho e sem corrimento vulvar. O diagnóstico é baseado no histórico e sinais clínicos, associados a exames complementares como hemograma e ultrassonografia (Trautwein et al., 2017).

O tratamento de escolha para piometra é a ovariosalpingohisterectomia ( $\mathrm{OSH})$, contudo, nos casos em que o tutor deseja que a cadela reproduza, o tratamento conservador através do uso de medicamentos pode ser indicado. Esse pode ser realizado através da administração de estrógenos, andrógenos, quinino, ocitocina e alcalóides derivados do ergot (Macente et al., 2016). Dessa forma, este trabalho tem como objetivo relatar 15 casos de piometra levando em consideração aspectos clínicos, laboratoriais e cirúrgicos em cadelas atendidas em uma clínica veterinária de Caxias do Sul.

\section{Metodologia}

Realizou-se um estudo retrospectivo através da análise de prontuários clínicos de uma clínica veterinária particular de Caxias do Sul. Foram inclusos na pesquisa apenas prontuários de cadelas diagnosticadas com piometra e excluídos prontuários com outros diagnósticos. Foram coletados e avaliados dados clínico-epidemiológicos como raça, porte, idade, histórico clínico, sinais clínicos, exames complementares e classificação da piometra, assim como o tratamento de escolha. Os animais foram agrupados quanto à raça como com ou sem raça definida, assim como referente ao seu porte como pequeno, médio ou grande. Segundo a idade, foram classificados conforme a metodologia de Fighera e colaboradores (2008) como filhotes (animais com menos de um ano), adultos (de um a nove anos) e idosos (acima de 9 anos).

Conforme a descrição nos prontuários, os animais foram classificados quanto ao tipo de piometra como com cérvix aberta ou fechada. Ainda, foi analisado o uso de contraceptivos, sendo anotado como utilizado (U) ou não utilizado (NU). Além disso, também foram coletadas informações sobre o histórico clínico e sinais clínicos, sendo que, cada animal poderia apresentar um ou mais sinais clínicos. Exames complementares como hemograma e ultrassonografia também foram avaliados, assim como o tratamento realizado (ovariohisterectomia) e a técnica cirúrgica escolhida em cada caso. Por tratarse de um estudo retrospectivo, algumas informações não foram obtidas.

\section{Resultados e Discussão}

O diagnóstico das 15 cadelas estudadas foi realizado por meio do histórico clínico, exames clínicos (anamnese e exame físico) e ultrassonográficos. De acordo com Lima (2019) a ultrassonografia é um método muito eficiente para o diagnóstico definitivo de piometra. Nesta pesquisa, houve uma maior ocorrência de casos de piometra fechada (60\%) quando comparado aos casos de piometra aberta (Tabela 1). Conforme a literatura, a piometra aberta é a forma mais comum da doença e menos agressiva devido à facilidade de o tutor perceber sinais clínicos característicos do distúrbio no animal como a secreção vulvar (Volpato; Lopes, 2015). As pacientes diagnosticadas com piometra fechada apresentavam sinais clínicos como desidratação, anorexia, abdômen abaulado e algia abdominal. Devido ao acúmulo de secreção uterina, pode ocorrer dilatação palpável do órgão, que é inversamente proporcional ao grau de abertura da cérvix (Conrado, 2009). A dilatação uterina 
palpável foi observada em duas pacientes do estudo. A ultrassonografia foi o exame complementar de imagem de escolha na maioria dos casos, no qual foi possível observar corpo e cornos uterinos dilatados e ecogênicos, com paredes espessadas e conteúdo intraluminal. Também foram encontrados restos de células de tecidos mortos ou danificados (debris celulares). Em casos de piometra fechada, se faz necessário o uso do exame ultrassonográfico (Lima, 2019) para seu diagnóstico, uma vez que, em casos de piometra de cérvix fechada não ocorre secreção vulvar para ajudar na elucidação do caso, neste estudo, sete pacientes foram submetidas a exames de imagem, onde seis foram diagnosticadas com piometra fechada através do exame ultrassonográfico e apenas uma paciente apresentou piometra aberta.

Tabela 1 - Relação entre raça, porte, tipo de piometra e tratamento das 15 cadelas diagnosticadas com piometra.

\begin{tabular}{lllll}
\hline ID & Raça & Porte & Tipo de Piometra & Tratamento \\
\hline 1 & SRD & Grande & Piometra aberta & OSH \\
2 & Dogue brasileiro & Grande & Piometra aberta & OSH \\
3 & Dogue brasileiro & Grande & Piometra fechada & OSH \\
4 & Dogue brasileiro & Grande & Piometra aberta & OSH \\
5 & Dogue brasileiro & Grande & Piometra aberta & OSH \\
6 & SRD & Médio & Piometra fechada & OSH \\
7 & Poodle & Pequeno & Piometra fechada & OSH \\
8 & SRD & Médio & Piometra aberta & OSH \\
9 & SRD & Grande & Piometra aberta & OSH \\
10 & Shihtzu & Pequeno & Piometra fechada & OSH \\
11 & Pinscher & Pequeno & Piometra fechada & OSH \\
12 & SRD & Pequeno & Piometra fechada & NI \\
13 & Maltês & Pequeno & Piometra fechada & OSH \\
14 & Pinscher & Pequeno & Piometra fechada & OSH \\
15 & Spitz & Pequeno & Piometra fechada & OSH \\
\hline
\end{tabular}

*OSH: ovariosalpingohisterectomia. NI: Não informado - tratamento não foi realizado na clínica. ID: identificação do paciente. SRD: Sem raça definida. Fonte: Autores.

Quanto às raças das cadelas estudadas, $67 \%$ foram classificados com raça definida e 33\% classificados em sem raça definida (Tabela 1). Dentre os animais com raça definida, o Dogue Brasileiro foi o mais prevalente (27\%), seguido por Pinscher (13\%), Shih Tzu, Maltês e Spitz (7\%) e Poodle (6\%). Apesar de saber-se que algumas raças são predispostas à piometra como Schnauzer Miniatura, Terrier Escocês, São Bernardo, Golden Retriever, Collie e Rottweiler (Smith, 2006), diferem das observadas nesta pesquisa.

Em relação à faixa etária, 10 pacientes eram adultas $(66,6 \%)$ e cinco eram idosas $(33,3 \%)$, contudo esta doença pode acometer animais de todas as idades. Há maior predisposição para o desenvolvimento da patologia em animais mais velhos devido à repetida estimulação da progesterona na fase lútea e a exposição a repetidosciclos estrais (Hedlund, 2008). A piometra está classicamente associada ao diestro, período em que há alta secreção de progesterona pelos ovários, ou ao uso de progestágenos exógenos. Nesta fase o útero está sob o efeito da progesterona produzida pelos corpos lúteos ovarianos (Schäfer-Somi, 2015). A progesterona tem a função de estimular o crescimento das glândulas endometriais suprimindo a atividade endometrial, o que pode resultar em hiperplasia endometrial cística que leva ao acúmulo de líquido na luz do órgão. Geralmente, a piometra quando acomete animais jovens está relacionada à terapia com estrógeno e 
progesterona exógenos. Já a piometra que ocorrer em cadelas idosas acontece em razão da repetida estimulação da progesterona fase lútea (Melo et al., 2020). Segundo o relato dos tutores, nenhum dos animais havia recebido progestágenos exógenos.

Corrêa (2020) relata que os sinais clínicos mais comuns da piometra são letargia, anorexia, inapetência, polidipsia, poliúria, vômitos, diarreia, secreção vaginal e desidratação. Estes corroboram com os observados neste estudo, em que se evidenciou sinais clínicos de prostração (7 casos), anorexia (7) e secreção vulvar (5) com maior ocorrência (Tabela 2). Ainda foram descritos outros sinais clínicos como abdômen abaulado (2), algia abdominal (2), febre (1), uveíte bilateral (1) e mucosas hiperêmicas (1). Em alguns casos, os animais eram assintomáticos, observando-se alterações apenas no exame de ultrassonografia.

Tabela 2 - Principais sinais clínicos descritos nos prontuários clínicos das 15 cadelas diagnosticadas com piometra.

\begin{tabular}{llllll}
\hline ID & Prostração & Anorexia & Secreção vulvar & $\begin{array}{l}\text { Abdômen } \\
\text { abaulado }\end{array}$ & $\begin{array}{l}\text { Algia } \\
\text { abdominal }\end{array}$ \\
1 & - & - & + & - & - \\
2 & - & - & + & - & + \\
3 & + & + & - & - & - \\
4 & - & + & + & - & - \\
5 & - & - & + & - & - \\
6 & + & + & - & - & - \\
7 & + & - & - & + & - \\
8 & + & + & + & - & - \\
9 & + & + & + & - & - \\
10 & - & - & - & - & - \\
11 & - & - & - & - & - \\
12 & - & - & - & - & - \\
13 & + & + & - & - & + \\
14 & + & + & - & - & + \\
15 & - & - & - & & - \\
\hline
\end{tabular}

+: sinal clínico presente; -: sinal clínico ausente; ID: identificação do paciente. Fonte: Autores.

Pela piometra se tratar de uma urgência cirúrgica nem todos os pacientes obtiveram uniformidadenos exames pré-cirúrgicos, o que impossibilita a uniformidade das informações quanto as análises clínicas. Desta forma neste presente estudo foram revisados 13 hemogramas, sendo este um dos exames complementares para avaliar o prognostico desses pacientes (Cyrino, 2020).

$\mathrm{Na}$ análise dos hemogramas realizados (Tabela 3) foram evidenciados quatro quadros de policitemia onde dois desses apresentaram PPT (proteínas plasmáticas totais) acima dos valores de referência, podendo ser classificados como policitemia relativa partindo do ponto que estes animais se apresentavam desidratados clinicamente. Em contraponto, em outros quatro exames revisados, foram evidenciados quadros de anemia classificados como anemia normocítica normocrômica, pelos índices hematimétricos, VCM (volume corpuscular médio) e CHCM (concentração da hemoglobina corpuscular média), além dos valores de eritrócitos, hematócrito e hemoglobina abaixo dos valores de referência. Este tipo de anemia está associado a vários processos inflamatórios incluindo infecções traumatismos e neoplasias. A ocorrência 
dessa anemia causada por doença inflamatória é multifatorial e inclui mudanças na homeostase do ferro, proliferação anormal das células progenitoras eritroides, produção de eritropoetina e redução do tempo de vida dos eritrócitos, além da resposta imunológica que ativa as células $\mathrm{T}$, produção de citocinas e interleucinas que afetam o metabolismo do ferro (Thrall, 2015). Nos demais exames revisados não houve alteração no eritrograma.

$\mathrm{Na}$ análise do leucograma destes pacientes (Tabela 3), foram evidenciadas cinco leucocitoses com desvio à esquerda regenerativo, sendo que dois destes foram caracterizados com a presença de neutrófilos tóxicos, que juntamente com a presença de células imaturas são critérios confiáveis para indicar processos infecciosos (Campbell, 2015). Além destes, outros dois hemogramas evidenciaram neutrófilos tóxicos na análise morfológica, porém estes não constavam com leucocitose. As alterações tóxicas nos neutrófilos são associadas a respostas inflamatórias compatíveis com o caso e segundo Thrall (2015) trata-se de uma alteração morfológica atribuída à produção alterada dos neutrófilos na medula óssea. Estes mesmos neutrófilos tóxicos também foram citados em outros dois leucogramas e também não evidenciavam leucocitose. Os demais oito exames não apresentaram leucocitose, devido a migração das células inflamatórias para a região acometida e, além disso, o leucrograma dentro dos valores de referência para a espécie estão associados a casos de piometra de cérvix aberta (Torres et al., 2019) corroborando com os achados deste estudo, onde três destes pacientes apresentaram o quadro de piometra de cérvix aberta.

Ainda na análise dos hemogramas, foram evidenciados quatro casos de trombocitose, um caso de trombocitopenia e os demais exames avaliados estavam dentro dos valores de referência para a espécie. A trombocitopenia pode ocorrer na piometra em razão a septicemia ou outros fatores como os efeitos diretos de bactérias nas plaquetas ou danos vasculares devido a reações imunes (Feldman, 1989). Já a trombocitose pode ser desencadeada pelos mecanismos existentes no aumento de plaquetas que estão relacionados a processos inflamatórios mediados pelas citocinas, principalmente a IL - 6 que estão envolvidas com a estimulação da trombopoiese (Rocha et al., 2019). Os dados detalhados de cada paciente foram expressos na Tabela 3.

Tabela 3 - Valores dos constituintes do eritrograma, das plaquetas, das proteínas plasmáticas totais e doleucograma das 15 cadelas diagnosticadas com piometra.

\begin{tabular}{|c|c|c|c|c|c|c|c|c|c|c|c|c|}
\hline Pacientes & Eri & $\mathrm{Hb}$ & $\mathrm{Ht}$ & $\mathrm{VCM}$ & $\mathrm{CHCM}$ & PPT & Plaquetas & $\begin{array}{l}\text { Leu } \\
\text { Totais }\end{array}$ & Neut & Bast & Linf & Monó \\
\hline 1 & NI & NI & NI & NI & NI & NI & NI & NI & NI & NI & NI & NI \\
\hline 2 & 1,83 & 14,2 & 14,0 & 76,5 & 30,0 & 6,6 & 225.000 & 6,600 & 5.808 & 0 & 660 & 132 \\
\hline 3 & 6,06 & 14,4 & 43,2 & 71,4 & 33,3 & 6,5 & 531 & 19.400 & 13.500 & 194 & 2.910 & 970 \\
\hline 4 & 5,65 & 13,6 & 41,9 & 74,2 & 32,5 & 8,6 & 51.500 & 15.500 & 9765 & 0 & 4185 & 1085 \\
\hline 5 & 5,01 & 11,2 & 34,4 & 68,66 & 22,56 & NI & 428.000 & 47.500 & 23.750 & 9.025 & 7.600 & 6.650 \\
\hline 6 & 5,49 & 11,9 & 34,9 & 63,6 & 34,0 & 9,0 & 100 & 25.900 & 9.324 & 8.806 & 4.662 & 2.590 \\
\hline 7 & 7,89 & 16,8 & 58,3 & 73,9 & 28 & 8,6 & 275 & 9.300 & 6.696 & 93 & 2.046 & 465 \\
\hline 8 & 5,97 & 14,1 & 41,6 & 69,7 & 33,9 & 7,9 & 418.000 & 10.700 & 8667 & 107 & 1070 & 428 \\
\hline 9 & NI & NI & NI & NI & NI & NI & NI & NI & NI & NI & NI & NI \\
\hline 10 & 7,15 & 16,3 & 53 & 74,13 & 30,75 & 7,00 & 471 & 8.900 & 5696 & 0 & 1958 & 801 \\
\hline 11 & 7,53 & 17,8 & 55 & 73,04 & 32,36 & 7,80 & 365 & 10.300 & 9601 & 0 & 2163 & 1030 \\
\hline 12 & 6,28 & 15,6 & 45,6 & 72,61 & 34,2 & $\mathrm{NI}$ & 216 & 13,30 & 9443 & 399 & 1862 & 1596 \\
\hline 13 & 9,0 & 21,0 & 64,0 & 71,1 & 32,8 & 7,2 & 260.000 & 11.500 & 8050 & 0 & 2630 & 116 \\
\hline 14 & 8,10 & 16,7 & 61,0 & 63,0 & 32,7 & 9,0 & 546.000 & 38.000 & 26220 & 3420 & 6840 & 1140 \\
\hline 15 & 3,32 & 8,75 & 25 & 75,3 & 35 & 11,50 & 789 & 36.300 & 29403 & 1452 & 4719 & 363 \\
\hline
\end{tabular}




\section{*NI: não informado. Fonte: Autores.}

Assim como nos exames de avaliação hematológica, nem todos os pacientes obtiveram uniformidade nos resultados dos exames da avaliação bioquímica (Tabela 4). Em relação à fosfatase alcalina (FA) quatro exames acima dos valores de referência foram encontrados. O aumento significativo de FA não sugere especificamente que há danos nos hepatócitos, mas pode ser resultante de colestase intra-hepática devido à baixa na atividade da alanina aminotransferase (ALT), ou decorrente ao aumento desta enzima em razão de corticoides endógenos causados pela dor e estresse no quadro infeccioso (Trautwein et al., 2017). Na avaliação de ALT dois casos se mostraram abaixo dos níveis de referência, e o restante dos casos estava dentro dos padrões. A baixa na atividade sérica daALT pode ser causada pela inibição da síntese da enzima no fígado em razão da ação das endotoxinas de bactérias, portanto ocorre uma diminuição das enzimas disponíveis para vazamento na circulação (Conrado, 2009).

Tabela 4 - Valores dos constituintes do exame bioquímico das 15 cadelas diagnosticadas com piometra.

\begin{tabular}{llllll}
\hline Pacientes & FA & Ureia & ALT & Creatinina & Fosforo \\
\hline 1 & NI & NI & NI & NI & NI \\
2 & NI & 391,0 & NI & 10,89 & 14,8 \\
3 & NI & NI & NI & NI & NI \\
4 & NI & NI & NI & NI & NI \\
5 & 209 & 20 & 83,00 & 1,00 & NI \\
6 & NI & 39,0 & 10,0 & 1,39 & NI \\
7 & 240,0 & 15,0 & 28,0 & 0,62 & NI \\
8 & 136,0 & 41,0 & 47,0 & 0,65 & NI \\
9 & NI & NI & NI & NI & NI \\
10 & 143 & 30,0 & 53,00 & 0,65 & NI \\
11 & 560 & NI & 68,00 & 0,54 & NI \\
12 & NI & NI & NI & 1,20 & NI \\
13 & 118 & 26,0 & 16,0 & 0,76 & NI \\
14 & 716,0 & 224,0 & 57,0 & 3,00 & NI \\
15 & NI & NI & NI & NI & NI \\
\hline
\end{tabular}

*NI: não informado. ID: identificação do paciente. Fonte: Autores.

Ainda na avaliação bioquímica, foram observados diferentes valores de ureia e creatinina (Tabela 4). Sobre a ureia, apenas dois casos estavam fora dos valores de referência, contudo, de diferentes formas, um caso estava acima dos valores e outro caso estava abaixo dos valores de referência. Já a creatinina dois casos estavam com valores acima do padrão. A avaliação de ureia e creatinina tem valor muito importante para o prognósticoda piometra. O aumento dos níveis séricos de ureia e creatinina podem estar relacionados a alguns fatores importantes como desidratação, redução da perfusão renal ou toxemia e a causas extra-renais em razão do catabolismo proteico associado com processos infecciosos graves (Trautwein et al., 2017), corroborando com os achados do presente estudo onde a paciente com ureia aumentada também apresentava quadro de desidratação.

O tratamento de piometra deve ser realizado de forma rápida e assertiva. Em relação ao tratamento, 14 casos foram tratados no local e apenas em um caso o tutor optou por realizar o tratamento em diferente local. O tratamento de escolha foi 
a OSH (técnica das 3 pinças) (Tabela 1) que é o tratamento de eleição para o distúrbio (Cyrino, 2020). As pacientes que se encontraram estáveis foram encaminhadas a cirurgia logo após o diagnóstico, sendo realizada antibioticoterapia transoperatória. Já as pacientes desidratadas foram submetidas a estabilização prévia com fluidoterapia e antibioticoterapia. Contudo, ainda é possível realizar o tratamento clínico através administração intravenosa de fluidos, remoção do conteúdo uterino e antibioticoterapia, a qual deve ser baseada no resultado da cultura e antibiograma do exsudato uterino (Barsanti, 2006).

No pós-operatório dos animais estudados, foi administrada antibioticoterapia e analgésicos. A castração das fêmeas por meio da OSH se destaca como o melhor método para prevenir a piometra, assim como outras doenças que podem afetar o sistema reprodutor das fêmeas (Silveira et al., 2013).

\section{Conclusão}

A piometra é uma patologia que acomete cadelas de meia idade a idosas, sem predileção por raça e que está relacionada aos hormônios controladores do cio e infecções bacterianas secundárias. Neste estudo houve uma predominância de casos de piometra fechada, sendo esta a forma mais grave da patologia. É importante ressaltar o uso de exames complementares como hemograma e ultrassonografia para o desfecho de cada caso, assim como a importância da cirurgia de OSH para o tratamento.

\section{Referências}

Barsanti, J. A. (2006). Genitourinary infections. Infectious diseases of the dog and cat, 1, 1376.

Campbell, T. W. (2015). Bioquímica clínica dos peixes e anfíbios. Hematologia e bioquímica clínica veterinária, 2, 529-535.

Conrado, F. (2009). Aspectos clínico-patológicos da piometra. Monografia (Graduação em Medicina Veterinária) - Faculdade de Veterinária, Universidade Federal do Rio Grande do Sul, (1) 1-78.

Corrêa, T. M., \& Oliveira, A. R. C. (2020). Avaliação ultrassonográfica da hiperplasia endometrial cística piometra em cadelassenis apóstratamento com farmacoterapia específica - relato de três casos. Revista Panorâmica, 72-97.

Cyrino, M. A. (2020). Biomarcadores inflamatórios e do estresse oxidativo na piometra canina. Dissertação (Programa de Pós-graduação em Biotecnologia Animal da Faculdade de Medicina Veterinária e Zootecnia) - Faculdade de Medicina Veterinária e Zootecnia da Universidade Estadual Paulista "Júlio de Mesquita Filho", Campus de Botucatu, 1-82.

Ettinger, S. J., \& Feldman, E. C. (2004). Tratado de medicina interna veterinária: doenças do cão e do gato. Afecções do estômago. 5ed. Rio de Janeiro: Guanabara Koogan, 1583-1614.

Fighera, R. A., Souza, T. M., Silva, M. C., Brum, J. S., Graca, D. L., Kommers, G. D., Irigoyen, L. F., \& Barros, C. S. (2008). Causes of death and reasons for euthanasia in dogs from the midland region of the Midwest of Rio Grande do Sul State, Brazil (1965-2004). Pesquisa Veterinária Brasileira, 28(4), 223-230.

Fossum, T. W., \& Macphail, C. (2019). Surgery of the Reproductive and Genital Systems. In: Small Animal Surgery. (5a ed.), Glendale: Elsevier. Cap 26, 720787.

Hedlund, C. S., \& Fossum, T. W. (2008). Cirurgia do sistema digestório. Cirurgia de pequenos animais, 3:339- 530.

Lima, J. W. G. (2019) Complexo hiperplasia endometrial cística-piometra: relato de caso em cadela. Monografia (Graduação em Medicina Veterinária) Universidade Federal Rural de Pernambuco, 1-29.

Macente, B., Gutierrez, R. R., Apparício, M., Mansano, C. F. M., Padilha, L. C., Tiosso, C. F., \& Toniollo, G. H. (2016). Uso da aglespristone no tratamento de piometra em cadela com gestação no estro subsequente. Investigação, 15(1):46-48.

Melo, P. T. A., de Andrade, L. A. C., \& Garcia, E. F. V. (2020). Perfil Clínico-Epidemiológico De Cadelas Com Piometra No Munícipio De Boa Vista-RR. Brazilian Journal of Animal and Environmental Research, 3(3):2230-2234.

Nelson, R. W., \& Couto, C. G. (2015). Condições Clínicas da Cadela e da Gata. In: Medicina Interna de Pequenos Animais. (5a ed.), Cap 57, $2633-2715$.

Rocha, M. N. A., Rocha, M. C. D. S., Kavasaki, M. L., Rodrigues, J. Y., Souza, W. F. D., \& Mendonça, A. J. (2019). Trombocitose: um estudo retrospectivo em 573 cães (2016-2017). Ciência Animal Brasileira, (20):1-10.

Silveira, B. C. P., Machado, E. A. A., Silva, W. M., Marinho, T. C. M. S., Ferreira, A. R. A., Bürger, C. P., \& Costa Neto, J. M. D. (2013). Estudo retrospectivo de ovariossalpingo-histerectomia em cadelas e gatas atendidas em Hospital Veterinário Escola no período de um ano. Arquivo Brasileiro de Medicina Veterinária e Zootecnia, 65(2), 335-340. 
Research, Society and Development, v. 10, n. 9, e35110918004, 2021

(CC BY 4.0) | ISSN 2525-3409 | DOI: http://dx.doi.org/10.33448/rsd-v10i9.18004

Smith, F. O. (2006). Canine pyometra. Theriogenology, 66(3), 610-612.

Thrall, M. A., Weiser, G., \& Allison, R. W. (2015). Veterinary hematology and clinical biochemistry. Maryland: Lippincott Williams \& Wilkins, 1, 518.

Torres, S, S., de Castro, N. L. M., da Silva, R. S., Wolkmer, P., Siqueira, L. C., \& Bassuino, D. M. Estudo retrospectivo de alterações hematológicas em casos de piometra canina. XXIV Seminário interinstitucional de ensino, pesquisa e extensão. 1-9.

Trautwein, L. G. C., Sant'anna, M. C., Justino, R. C., Giordano, L. G. P., Flaiban, K. K. M. D. C., \& Martins, M. I. M. (2017). Piometras em cadelas: relação entre o prognóstico clínico e o diagnóstico laboratorial. Ciência Animal Brasileira, 18, 1-10.

Volpato, R., \& Lopes, M. D. (2015). Fatores envolvidos nos mecanismos de abertura cervical em cadelas com piometra. Veterinária e Zootecnia, 22(3), 335346.

Schäfer-Somi, S. (2015). Common uterine disorders in the bitch: challenges to diagnosis and treatment. Revista Brasileira de Reprodução Animal, 39(1), 234239. 